\title{
Pancreatic Cancer: Multicenter Prospective Data Collection and Analysis by the Hungarian Pancreatic Study Group
}

\author{
Gábor Lakatos ${ }^{1}$, Anita Balázs², Balázs Kui², Szilárd Gódi ${ }^{3}$, Ákos Szücs ${ }^{4}$, Andrea Szentesi ${ }^{2}$, Zsolt Szentkereszty ${ }^{5}$, \\ Richárd Szmola ${ }^{6}$, Dezső Kelemen ${ }^{7}$, Róbert Papp ${ }^{7}$, Áron Vincze ${ }^{3}$, József Czimmer ${ }^{3}$, Gabriella Pár ${ }^{3}$, Judit Bajor ${ }^{3}$, Imre \\ Szabó $^{3}$, Ferenc Izbéki ${ }^{8}$, Adrienn Halász ${ }^{8}$, László Leindler ${ }^{9}$, Gyula Farkas Jr. ${ }^{9}$, Tamás Takács ${ }^{2}$, László Czakó ${ }^{2}$, Zoltán Szepes ${ }^{2}$, \\ Péter Hegyi ${ }^{2,10,11^{*}}$, Zsuzsanna Kahán ${ }^{12, *}$
}

\footnotetext{
1) Dept. Oncology, St. Istvan and St. Laszlo Hospital and OutPatient Department, Budapest; 2) $1^{\text {st }}$ Dept. Medicine, University of Szeged; 3) $1^{\text {st }}$ Dept. Medicine, University of Pécs; 4) $1^{\text {st }}$ Dept. Surgery, Semmelweis University, Budapest; 5) Institute of Surgery, Clinical Center, University of Debrecen; 6) Dept. Interventional Gastroenterology, National Institute of Oncology, Budapest; 7) Dept. Surgery, University of Pécs; 8) $1^{\text {st }}$ Dept. Medicine, Szent György University Teaching Hospital of County Fejér, Székesfehérvár; 9) Dept. Surgery, University of Szeged; 10) Hungarian Academy of Sciences, University of Szeged, Momentum Gastroenterology Multidisciplinary Research Group; 11) Institute for Translational Medicine \& 1st Department of Medicine, University of Pécs; 12) Dept. Oncotherapy, University of Szeged, Hungary
}

\section{Address for correspondence: Prof. Dr. Peter Hegyi University of Pécs; Centre for Translational Medicine; Pécs, Hungary hegyi2009@gmail.com}

Received: 11.12.2015 Accepted: 26.02.12016

*the last two authors equally contributed to this work

\begin{abstract}
Background \& Aims: Pancreatic cancer is a devastating disease with poor prognosis. There is very limited information available regarding the epidemiology and treatment strategies of pancreatic cancer in Central Europe. The purpose of the study was to prospectively collect and analyze data of pancreatic cancer in the Hungarian population.

Methods: The Hungarian Pancreatic Study Group (HPSG) organized prospective, uniform data collection. Altogether 354 patients were enrolled from 14 Hungarian centers.

Results: Chronic pancreatitis was present in $3.7 \%$ of the cases, while $33.7 \%$ of the patients had diabetes. Family history for pancreatic cancer was positive in $4.8 \%$. The most frequent presenting symptoms included pain $(63.8 \%)$, weight loss (63\%) and jaundice (52.5\%). The reported frequency of smoking and alcohol consumption was lower than expected $(28.5 \%$ and $27.4 \%$, respectively). The majority of patients $(75.6 \%)$ were diagnosed with advanced disease. Most patients (83.6\%) had a primary tumor located in the pancreatic head. The histological diagnosis was ductal adenocarcinoma in $90.7 \%$ of the cases, while neuroendocrine tumor was present in 5.3\%. Biliary stent implantation was performed in 166 patients, $59.2 \%$ of them received metal stents. Primary tumor resection was performed in 60 (16.9\%) patients. Enteral or biliary bypass was done in 35 and 49 patients, respectively. In a multivariate Cox-regression model, smoking status and presence of gemcitabine-based chemotherapy were identified as independent predictors for overall survival.

Conclusion: We report the first data from a large cohort of Hungarian pancreatic cancer patients. We identified smoking status and chemotherapy as independent predictors in this cohort.
\end{abstract}

Key words: pancreatic cancer - Hungarian cohort - acute pancreatitis - chronic pancreatitis - diabetes mellitus.

Abbreviations: EBM: evidence-based medicine; EPI: exocrine pancreatic insufficiency; FNAB: fine needle aspiration biopsy; HPSG: Hungarian Pancreatic Study Group; MDCT: multi-detector computed tomography; OS: overall survival; PC: pancreatic cancer; RPP: Registry for Pancreatic Patients

\section{INTRODUCTION}

Pancreatic cancer (PC) is one of the most aggressive types of human malignancies and a major health problem. Pancreatic cancer is a relatively rare type of tumor, but due to its high mortality rate it is one of the most frequent causes of cancer death in the world.[1] In developed countries the incidence of $\mathrm{PC}$ is the seventh, and the mortality rate is the fifth among all cancers [2]. In Hungary both the incidence and mortality are one of the highest in Europe. There were 2,373 newly diagnosed PC patients in Hungary in 2010, and 1,837 died due to the disease [3].

The number of patients with PC is increasing globally. It is estimated that in 2012 there were 103,773 newly diagnosed cases and 104,463 fatal outcomes in Europe [4]. There are no screening tests for early detection of PC. By the time of diagnosis, less than $15 \%$ of patients can be offered a potential curative treatment, and up to $30 \%$ of the patients die within 12 months [5]. 
Data suggest that the onco-epidemiological situation related to pancreatic cancer in Central European countries is even worse compared to that in the Western world [6]. An epidemiologic analysis from 51 countries across the world has shown the highest mortality rates in Central Europe with trends towards increasing mortality in Romania, along with Albania, Spain and Croatia [7]. An epidemiologic study conducted in Serbia between 1991 and 2010 demonstrated high mortality rates with increasing mortality trend in both genders and in most age groups [8].

There is only limited information available on the management of PC from Central Europe including Hungary. In order to improve outcome of $\mathrm{PC}$, it is essential to determine which factors contribute to the unfavorable trends seen in less developed countries. The main purpose of our study was to collect information regarding the diagnosis, management and outcome of pancreatic cancer in Hungary. Here we present the first large prospectively collected and analyzed data of pancreatic cancer in the Hungarian population.

\section{PATIENTS AND METHODS}

The Hungarian Pancreatic Study Group (HPSG) was established in 2011 in order to improve the care of patients suffering from pancreatic diseases. To achieve our aims we (i) developed an electronic data registry for patients (www. pancreas.hu), (ii) published the currently available evidencebased medicine (EBM) guidelines [9-13], (iii) established specific study sessions including the pancreatic cancer one, (iv) and organized multicenter clinical trials [14-17].

For this study the HPSG collected data from patients diagnosed with PC between September 2012 and March 2014 using uniform questionnaire and clinical data sheets. Patients were enrolled from 14 Hungarian centers including endoscopy units, gastroenterological, oncological and surgical departments. The characteristics of the single departments and the number of patients enrolled by each center are summarized in Table I.

Demographic data, data of possible risk factors, symptoms, diagnosis, staging, therapy and survival were assessed. Data collection was performed using a web-based electronic data collection method as part of the Registry for Pancreatic Patients (RPP).

Demographic data included age and gender of patients. Information about alcohol consumption and smoking (frequency and total amount of daily consumption), body mass index (BMI), history of acute and chronic pancreatitis, diabetes mellitus and familial PC has been collected as possible risk factors. Frequency of symptoms and clinical signs, such as fever, pain, diarrhea, jaundice and weight loss were also evaluated.

Cancer related data included the date of diagnosis, extension of the disease, location of the primary tumor, histological type, the method used to obtain histological diagnosis and the level of CA 19-9 at the time of diagnosis. Diagnosis and staging of pancreatic cancer was based on imaging tests including multi-detector computed tomography (MDCT), magnetic resonance imaging (MRI), endoscopic retrograde cholangiopancreatography (ERCP) and endoscopic ultrasonography (EUS). Primary resectable tumor, locally advanced and metastatic disease were distinguished. Histological diagnosis was performed using brush cytology during ERCP, fine needle aspiration biopsy (FNAB) or surgical biopsy/resection.

The database included information on endoscopic, surgical, oncological and supportive therapy performed. The proportion of plastic or metal stents used for biliary drainage was determined. Information on the frequency of duodenal stent implantation was also recorded. Data on surgical resection (including margin status; R0, R1, R2) has been collected for patients with a resectable primary tumor. Palliative biliary and enteral bypass were recorded as well. If a patient received oncological treatment (radiation therapy or chemotherapy) for PC, the type and intent (neo-adjuvant, adjuvant, palliative) of therapy and the name of the chemotherapeutic agent used

Table I. Characteristics of the participating centers

\begin{tabular}{llcc}
\hline Name of institution & Type of institution & Department profile & $\begin{array}{c}\text { Number of patients } \\
\text { enrolled (n) }\end{array}$ \\
\hline Borsod-Abaúj-Zemplén County General Hospital, Miskolc & General Hospital & Gastroenterology & 2 \\
Bács-Kiskun County Municipality Hospital, Kecskemét & General Hospital & Gastroenterology & 2 \\
Pándy Kálmán Békés County Hospital, Gyula & General Hospital & Gastroenterology & 11 \\
Department of Interventional Gastroenterology, National & National Institution & Endoscopy Unit & 27 \\
Institute of Oncology, Budapest & & & 18 \\
Institute of Surgery, Clinical Center, University of Debrecen & University Hospital & Surgery & 41 \\
Department of Surgery, University of Pécs & University Hospital & Surgery & 29 \\
First Department of Medicine, University of Pécs, Hungary & University Hospital & Gastroenterology & 89 \\
First Department of Internal Medicine, University of Szeged & University Hospital & Gastroenterology & 7 \\
Second Department of Internal Medicine, University of Szeged & University Hospital & Gastroenterology & 18 \\
Department of Oncotherapy, University of Szeged & University Hospital & Oncology & 55 \\
Department of Surgery, University of Szeged & University Hospital & Surgery & 43 \\
First Department of Medicine, Szent György University Teaching & General Hospital & Gastroenterology & 2 \\
Hospital of County Fejér, Székesfehérvár & & & 10 \\
First Department of Surgery, Semmelweis University, Budapest & University Hospital & Surgery & Gastroenterology \\
Dr. Réthy Pál Hospital, Békéscsaba & General Hospital & & \\
\hline
\end{tabular}


were also noted. Data collected on supportive therapy consisted of pancreatic enzyme replacement, pain control and the management of diabetes mellitus.

Information on survival status was obtained from the Hungarian Central Statistical Office. Survival was defined as the number of months between date of diagnosis and date of death (if known).

The research involved human participants. All data have been collected after the patients had given written informed consent. The study was approved by the Secretary of Medical Research Council, Scientific and Research Ethics Committee, approval number 22254-1/2012/EKU (391/PI/2012).

\section{Statistical analysis}

Statistical analysis with one-way ANOVA and Student $t$-test was performed. Survival data was analysed by plotting Kaplan-Meier curves and LogRank test. A multivariate Coxregression analysis was performed to identify independent predictors of overall survival. Variables with a $p$ value of $<0.2$ were included in the Cox-regression analysis, in addition gender and location of the tumor were added as arbitrary variables. Values are expressed as means \pm standard deviation (SD) if not stated otherwise. A P value $<0.05$ was considered statistically significant.

\section{RESULTS}

Three hundred fifty-four patients were enrolled into the study. Mean age of the population was 65.2 years (SD 11.5, range: $23-88$ years). There were more males than females ( $53.4 \%$ vs. $46.6 \%$, respectively).

\section{Risk factors}

One hundred and one patients $(28.5 \%)$ were recorded as smoking regularly. Twenty-eight patients (7.9\%) were smoking more than 20 cigarettes per day. Alcohol consumption was reported in 97 patients $(27.4 \%)$, whereas 44 (12.4\%) were drinking alcohol on a daily basis. Data on BMI at the time of diagnosis was available for 297 patients (83.9\%). The time of diagnosis was defined as the date of the first imaging modality (CT scan, MRI or ERCP) performed giving the diagnosis of pancreatic cancer. One hundred and seventy-one (57.6\%) patients had a normal BMI (normal range: $18.5-24.99 \mathrm{~kg} / \mathrm{m}^{2}$ ), while 103 (34.6\%) were overweight (BMI: 25.0-29.99 kg/m²) and $23(7.7 \%)$ were obese $\left(\mathrm{BMI} \geq 30.0 \mathrm{~kg} / \mathrm{m}^{2}\right)$.

Only 8 patients $(2.3 \%)$ had a positive history for recurrent acute pancreatitis, 13 (3.7\%) were diagnosed with chronic pancreatitis. Approximately one third of the population $(n=119,33.7 \%)$ had diabetes; almost half $(n=57,47.9 \%)$ of them were using insulin. Positive family history for pancreatic cancer was found in 13 patients (3.6\%).

\section{Symptoms and signs}

The most frequent symptoms at the time of diagnosis were abdominal pain and weight loss (unexplained loss of more than $5 \%$ of the body weight within six month), which were present in $63.8 \%$ and $63 \%$ of all patients. Jaundice (bilirubin concentration higher than $35 \mu \mathrm{mol} / \mathrm{L}$ ) was found in $52.5 \%$. Interestingly, there was only a small difference in the frequency of jaundice between patients having a tumor in the pancreatic head (53.1\%) and those having the cancer in the body or tail (50\%). Diarrhea was recorded in $13.8 \%$ of the patients, $7.9 \%$ had fever. Newly diagnosed diabetes was found in $2.3 \%$ of the studied population. The cancer was recognized accidentally in $6.5 \%$; these patients were symptom free. Presenting symptoms are summarized in Fig. 1.

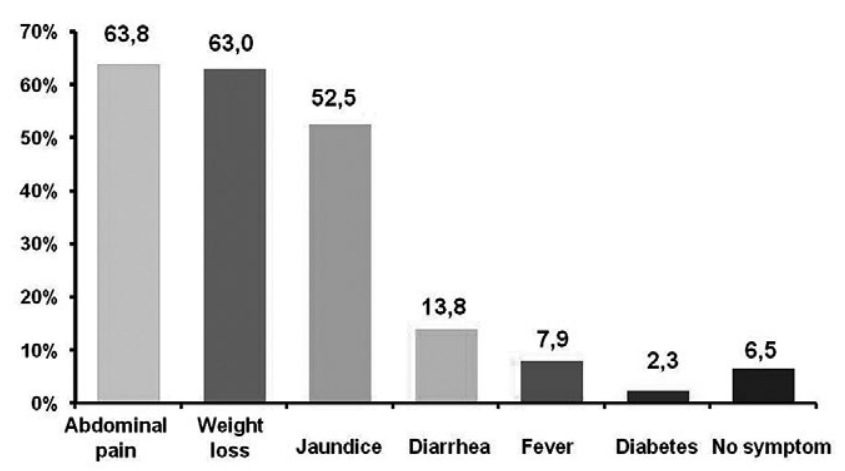

Fig. 1. Incidence of symptoms and clinical signs at the time of diagnosis.

\section{Cancer related data}

Information on tumor stage was missing in 29 cases (8.2\%). Seventy-nine patients $(24.3 \%)$ had resectable disease. The tumor was located to the pancreatic head in the majority (77.2\%) of resectable cases $(n=61)$, while $7(8.9 \%)$ patients had cancer located in the body and $11(13.9 \%)$ in the tail of the pancreas. The proportion of locally unresectable and metastatic cancer at initial diagnosis is shown in Table II.

Table II. Distribution of pancreatic cancer cases by disease stage.

\begin{tabular}{lcc}
\hline Disease stage & Number of patients (n) & Percentage (\%) \\
\hline Resectable & 79 & 24.3 \\
Locally advanced & 138 & 42.4 \\
Metastatic & 108 & 33.2 \\
\hline
\end{tabular}

In the majority of cases $(n=285,80.5 \%)$ the primary tumor was located in the head of the pancreas. Cancer of the body and tail was found in 27 and 29 cases (7.6\% and $8.2 \%$, respectively). Tumor location was unknown in 13 cases $(3.7 \%)$.

Histological/cytological diagnosis was available for 227 patients $(64.1 \%)$. The diagnosis was obtained via image guided fine needle aspiration biopsy (59.7\%), brush cytology during ERCP (11.6\%), or surgical biopsy/resection (28.7\%). The biopsy revealed ductal adenocarcinoma in the majority of the cases $(n=206,90.7 \%)$. Adenocarcinoma of the papilla of Vater was confirmed in 5 cases $(2.2 \%)$, while 12 patients $(5.3 \%)$ had neuroendocrine carcinoma. There were 2 cases of intraductal papillary-mucinous carcinoma, one case of solid-pseudopapillary carcinoma and one unique case of histologically proven diffuse large B-cell lymphoma located in the pancreas.

Serum CA 19-9 level was investigated for ductal adenocarcinoma and Vater's papilla carcinoma $(n=211)$ at the time of initial diagnosis. Data were available for 83 patients. The level of CA 19-9 was elevated in 65 cases (78\%). 


\section{Therapy}

Biliary stent implantation during ERCP was performed in 166 cases. Metal stents were used more commonly than plastic ones (59\% vs. $40.1 \%$, respectively). Duodenal stent placement for small bowel obstruction was reported in only two cases.

From the 79 patients with a resectable primary tumor, 60 underwent surgical resection. The distribution of tumor location of surgical cases was consistent with data reported on resectable cases: head 50 (83.3\%), body 4 (6.7\%), tail 6 (10\%). Fifty patients had tumor free resection margins (R0), four patients had microscopic (R1) and six macroscopic (R2) residual disease. There is no information available why 19 patients with resectable pancreatic tumor did not undergo surgery. Palliative surgical treatment was performed in 84 cases. Thirty-five patients underwent enteral bypass, while biliary bypass reconstruction was performed in 49 cases (Table III).

Table III. Surgical treatment

\begin{tabular}{lcc}
\hline Disease stage & Type of surgery & Number of patients (n) \\
\hline \multirow{3}{*}{ Resectable $(\mathrm{n}=79)$} & Curative & 60 \\
& R0 resection & 50 \\
& R1 resection & 4 \\
Unresectable $(\mathrm{n}=246)$ & R2 resection & 6 \\
& Palliative & 84 \\
& Enteral bypass & 35 \\
& Biliary bypass & 49 \\
\hline
\end{tabular}

There is very limited data available in terms of the oncological treatment used in the studied population. Most information on oncotherapy originated from oncology departments presenting their cases. Only one patient was reported to have received radiotherapy with palliative intent for neuroendocrine carcinoma. Administration of chemotherapy was recorded in 42 cases. Nine patients received adjuvant treatment; palliative therapy was used in 33 cases. Every patient received gemcitabine-based chemotherapy. Neither the FOLFIRINOX regimen, nor nab-paclitaxel was used in this cohort of patients. There is no reported case in which neo-adjuvant chemotherapy was given.

Information about the use of analgesics was available in 179 patients $(50.6 \%)$. Regular intake of painkillers was found in 73 cases $(40.8 \%)$. Minor analgesics were needed for 56 patients $(31.3 \%)$, while 43 patients $(24 \%)$ were administered major analgesics for severe pain. It should be noted that $58.9 \%$ $(43 / 73)$ of the patients suffering from pain would have needed major analgesics.

Exocrine pancreatic insufficiency (EPI) affects the majority of patients with pancreatic cancer. Pancreatic enzyme replacement therapy is recommended to relieve EPI-related gastrointestinal symptoms. Data on enzyme replacement therapy is available for 311 patients. Supportive treatment of the studied population is summarized in Table IV.

\section{Survival}

Survival data is available for 194 patients. Survival was defined as the number of months between the date of diagnosis and date of death. Overall survival (OS) for the whole population was 8.7 months.
Table IV. Supportive treatment (some patients received both minor and major analgesics).

\begin{tabular}{lcc}
\hline $\begin{array}{l}\text { Type of therapy } \\
\text { (number of data available) }\end{array}$ & Number of patients (n) & Percentage (\%) \\
\hline Analgesics $(\mathrm{n}=179)$ & 73 & 40.8 \\
Minor & 56 & 31.3 \\
Major & 43 & 24 \\
Pancreatic enzyme & 52 & 16.7 \\
replacement $(\mathrm{n}=311)$ & & \\
\hline
\end{tabular}

Overall survival of patients with histologically proven ductal adenocarcinoma $(n=133)$ was $9.97 \pm 1.77$ months. Neuroendocrine carcinoma patients had a better prognosis with an OS of $14 \pm 5.21$ months. However, survival data is available for only 4 patients with a neuroendocrine tumor. There is no information available regarding the survival of patients with carcinoma of the papilla of Vater.

Overall survival of ductal adenocarcinoma patients was significantly different according to smoking habits (pLogRank=0.049, Fig. 2A) and for patients who have received gemcitabine based chemotherapy ( $p=0.013$, Fig. $2 B$ ) in a Kaplan-Meier analysis The number of curative surgical resections was low $(n=19)$, survival data were not available from 10 patients and OS was not analysed according to the surgical resection status. There was no association between gender, tumor stage, location, alcohol consumption, diabetes, presence of lymph node metastasis or BMI and OS. In a multivariate Cox-regression model, smoking status and presence of gemcitabine-based chemotherapy were identified as independent predictors for overall survival (Table V).

Table V. Overall survival of patients with pancreatic ductal adenocarcinoma according to gender, smoking status, alcohol consumption, location and chemotherapy.

\begin{tabular}{llll}
\hline & P-value & Hazard Ratio & 95\% CI \\
\hline Gender & 0.84 & & \\
Alcohol consumption & 0.15 & & \\
Smoking & 0.016 & & \\
$\quad$ Yes & 0.016 & 1.61 & $1.10-2.37$ \\
No & Reference & & \\
Location & 0.45 & & \\
Chemotherapy & 0.045 & & $1.15-3.77$ \\
$\quad$ No data & 0.016 & 2.08 & $1.07-2.88$ \\
No & 0.027 & 1.75 & \\
Yes & Reference & & \\
\hline
\end{tabular}

\section{DISCUSSION}

Recently, PC has shown an increasing trend in incidence rates among both men and women [18]. The number of cases of PC in Central Europe is also increasing, mortality rates in this region are among the highest in the world [7].

There is very limited data available about the management and outcome of PC in Hungary. In order to improve the prognosis of $\mathrm{PC}$, it is essential to determine which factors contribute to the high mortality rates seen in Central Europe. 

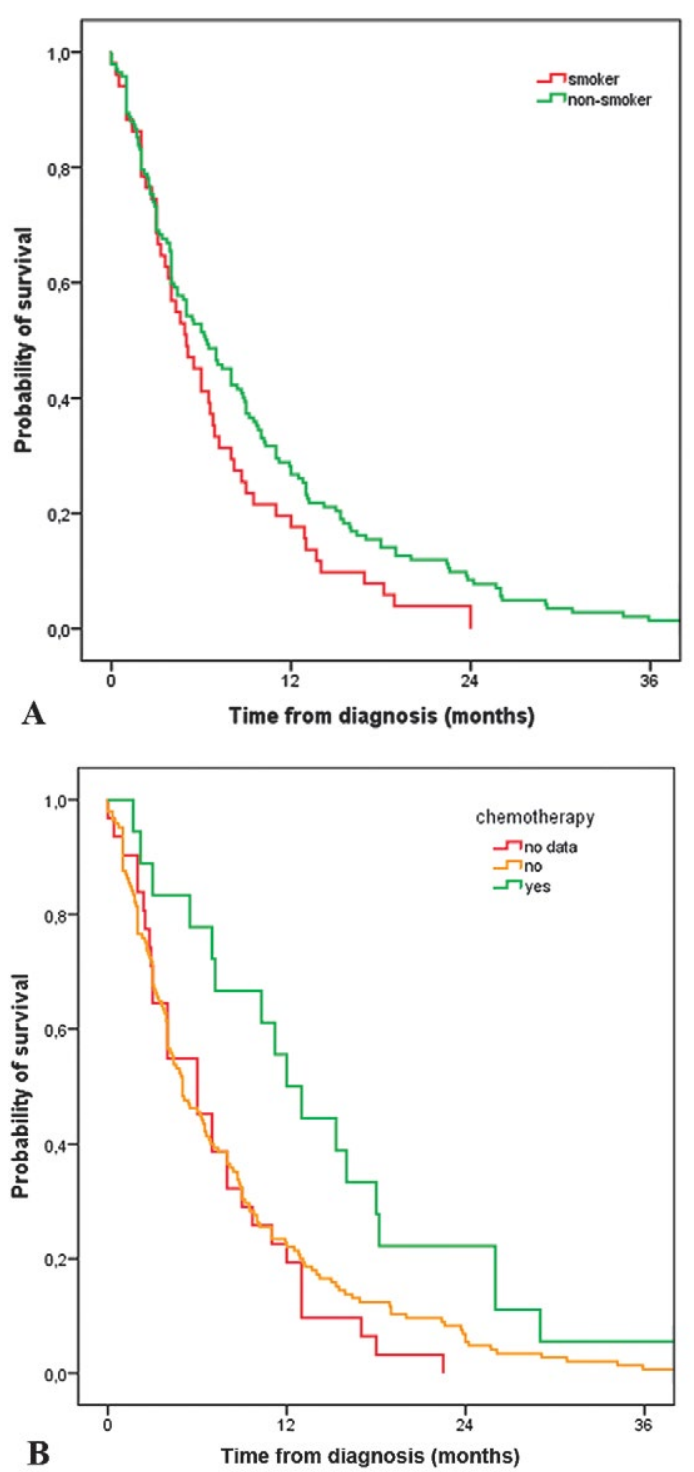

Fig. 2. Overall survival of patients with pancreatic ductal adenocarcinoma according to smoking status (A) and chemotherapy (B).

We report the first data from a large cohort of Hungarian PC patients. Patients were enrolled from departments with different profiles. Most of the data was provided by university centres or general hospitals with a teaching function. The proportion of patients enrolled by the smaller hospitals was low, which is a limitation of the study. Data were compared to results from published literature.

There are multiple risk factors possibly related to PC. Smoking counts as the strongest environmental risk factor for PC. A meta-analysis reported an elevated risk of PC both for current and former smokers [19]. In the Hungarian cohort $28.5 \%$ of patients were smoking regularly, which reflects the smoking habits in Hungary (28.9\% of the adult population were smoking in 2012 in Hungary) [20]. The rate of current smokers among patients with PC was the same as the average current smoker rate in the general population. These data do not support the relationship between smoking and the elevated risk of PC. Alcohol consumption contributes to episodes of acute pancreatitis, and is the most common cause of chronic pancreatitis. It seems reasonable that heavy alcohol consumption elevates the risk of PC. In our cohort regular consumption of alcohol was reported in $27.4 \%$ of the patients.

Chronic pancreatitis has been proposed as an independent risk factor for PC and explains about 3\% of the cases [21]. In our study, the prevalence of both acute recurrent and chronic pancreatitis was low. The rate of chronic pancreatitis was consistent with literature data. There were 13 patients with positive family history of PC; the youngest patient was 23 years old. Over the past decades multiple studies have reported a positive association between diabetes and PC [22]. However, as diabetes could be a manifestation of PC, the link between diabetes and the risk of cancer is controversial. New onset diabetes probably should be evaluated in a different manner than the diabetes lasting for more than 3 years. Hyperglycemia or manifest diabetes is present in $50-80 \%$ of patients diagnosed with PC. In our cohort, approximately one third of the patients had diabetes and half of them were using insulin at the time of diagnosis. There is no information about the duration of diabetes before the diagnosis of PC. Obesity and overweight have been shown to be risk factors for PC [22]. Most of the patients in our study had a normal BMI or were overweight.

Prevalence of the presenting symptoms was consistent with literature data. The most frequent symptoms at the time of diagnosis were abdominal pain and weight loss. The location of the tumor did not affect the prevalence of jaundice, which was present in more than half of the patients. New onset diabetes was found in only $2.3 \%$ of the studied population.

Histological diagnosis was not available in more than one third of the patients. In most cases histology was performed via image guided fine needle aspiration biopsy. In accordance with literature data [24], histology revealed ductal adenocarcinoma in the majority of the cases. Twelve patients had neuroendocrine carcinoma, while other subtypes were only found occasionally. In this study neuroendocrine carcinoma patients had a better prognosis than patients with ductal adenocarcinoma. Overall survival for the whole population was 8.7 months. The location of the tumor was predominantly in the head of the pancreas. Serum CA 19-9 level was found to be elevated in $78 \%$ of the cases with ductal adenocarcinoma and carcinoma of the papilla of Vater. The rate of false-negativity was $22 \%$, which confirms that CA 19-9 determination cannot be used as a screening test for the detection of pancreatic cancer [25].

About 15 to 20 percent of patients with PC have resectable disease at the time of diagnosis [26]. In this cohort more patients had resectable or locally advanced tumors $(24.3 \%$ and $42.4 \%$, respectively) as recorded in the literature. One third of the patients had metastatic disease at initial diagnosis. From the 79 patients with a resectable primary tumor, 60 had underwent surgical resection, most of them having R0 resection. Median survival following surgical resection ranges between 11.2 and 25.5 month [27]. Since OS data were available for only a few patients $(n=9)$ who had undergone surgical resection, survival data were not analysed according to the surgical resection status. Information is lacking why 19 patients with resectable pancreatic tumor did not undergo surgery. Inappropriate overall status or comorbidities may have been the cause that these patients were not eligible for surgery. 
Biliary obstruction (defined as extrahepatic obstruction of the common bile duct causing jaundice [bilirubin $>35$ $\mu \mathrm{mol} / \mathrm{L}])$ was seen in $52.5 \%$ of the patients. It is controversial whether metal or plastic stents should be used for biliary obstruction caused by PC. In a recent study the use of metal stents was associated with an outcome benefit [28]. In our cohort metal stents were used more frequently than plastic ones. Gastrointestinal obstruction was resolved via palliative enteral bypass surgery in most of the cases. Endoscopic stent placement was performed in only two cases, which can be explained by the fact that self-expandable enteral stents are not financially reimbursed, therefore not available at the majority of Hungarian endoscopy units.

Information about oncological therapy was only available from oncology department patients. There was no recorded administration of radiotherapy for patients with ductal adenocarcinoma, which reflects that radiotherapy is not used in routine clinical practice for PC in Hungary. Gemcitabine-based chemotherapy was administered in all cases. Although, the FOLFIRINOX regimen has shown significant survival benefit for selected patients in PC [29], there are only some centers in Hungary using this protocol; it is not used routinely. The nab-paclitaxel plus gemcitabine regimen has recently become a first-line treatment option for patients with metastatic PC [30]. Nab-paclitaxel is currently not available in Hungary. There was no association between gender, tumor stage, location, alcohol consumption, diabetes, presence of lymph node metastasis or BMI and overall survival. Smoking status and presence of gemcitabine-based chemotherapy were identified as independent predictors for overall survival.

Information on supportive therapy is missing in many cases. It should be emphasized that the majority of patients suffering from pain would be in need of the use of major analgesics. Exocrine pancreatic insufficiency (EPI) affects the majority of patients with pancreatic cancer. Pancreatic enzyme replacement therapy is recommended to alleviate EPI-related gastrointestinal symptoms and improve quality of life. In our cohort only $16.7 \%$ of the patients received pancreatic enzyme substitution.

\section{CONCLUSIONS}

In the Hungarian cohort, the frequency of both acute recurrent and chronic pancreatitis was low. Most patients had histologically proven ductal adenocarcinoma; the other histological subtypes were rare. Biliary stent placement is a routine intervention for the management of biliary obstruction occurring in PC. Hungarian endoscopists use more metal stents than plastic ones. In cases of enteral obstruction, palliative surgical bypass is the preferred approach compared with the endoscopic placement of duodenal stents. Smoking status and presence of gemcitabine-based chemotherapy were identified as independent predictors for overall survival. Future plans of the HPSG include improving the quality of data collection and the extension of the database to other Central and Eastern European countries.

Conflicts of interest: No conflict to declare.
Authors' contribution: P.H.: study concept; P.H. and A.S.: study design, manuscript preparation; G.L.: data analysis and interpretation, quality control of data, statistical analysis and manuscript preparation; A.B., B.K., A.G, A.S., A.S.: data analysis and interpretation: Z.H., A.S.: quality control of data. All authors contributed to data acquisition, and approved the final version of the manuscript.

Acknowledgements: The study was supported by the Hungarian Academy of Sciences - University of Szeged, Momentum Gastroenterology Multidisciplinary Research Group (960152 to Peter Hegyi) and the National Research, Development and Innovation Office (K116634 to Peter Hegyi). The above funding sources had no involvement in the study design, in the collection, analysis and interpretation of data nor in the writing of the report and in the decision to submit the article for publication. We thank Peter Lakatos MD (First Department of Internal Medicine, Semmelweis University, Budapest) for assisting in the statistical analysis.

\section{REFERENCES}

1. Jemal A, Bray F, Center MM, Ferlay J, Ward E, Forman D. Global cancer statistics. CA Cancer J Clin 2011; 61: 69-90. doi: 10.3322/ caac. 20107

2. Ferlay J, Soerjomataram I, Ervik M, et al. GLOBOCAN 2012 v1.0, Cancer Incidence and Mortality Worldwide: IARC CancerBase No. 11 [Internet]. Lyon, France: International Agency for Research on Cancer; 2013. Available from: http://globocan.iarc.fr. Accessed December 2013.

3. Registry HNC. In: Oncology NIo, editor.; 2010.

4. Ferlay J, Steliarova-Foucher E, Lortet-Tieulent J, et al. Cancer incidence and mortality patterns in Europe: estimates for 40 countries in 2012. Eur J Cancer 2013; 49: 1374-1403. doi: 10.1016/j.ejca.2012.12.027

5. Barugola G, Partelli S, Marcucci S, et al. Resectable pancreatic cancer: who really benefits from resection? Ann Surg Oncol 2009; 16: 3316 3322. doi: 10.1245/s10434-009-0670-7

6. Smiljana R, Mikov MM, Petrovic V, Jasna T, Tihomir D, Milanka T. Epidemiology of pancreatic cancer in vojvodina province in Serbia. Asian Pac J Cancer Prev 2014; 15: 10779-10782.

7. Hariharan D, Saied A, Kocher HM. Analysis of mortality rates for pancreatic cancer across the world. HPB (Oxford) 2008; 10: 58-62. doi: $10.1080 / 13651820701883148$

8. Ilic M, Vlajinac H, Marinkovic J, Kocev N. Pancreatic cancer mortality in Serbia from 1991-2010 - a joinpoint analysis. Croat Med J 2013; 54: 369-375. doi: $10.3325 / \mathrm{cmj} .2013 .54 .369$

9. Szmola R, Farkas G, Hegyi P, et al. Pancreatic cancer. Evidence based management guidelines of the Hungarian Pancreatic Study Group. Orv Hetil 2015; 156: 326-339. doi: 10.1556/OH.2015.30063

10. Párniczky A, Czakó L, Dubravcsik Z, et al. Pediatric pancreatitis. Evidence based management guidelines of the Hungarian Pancreatic Study Group. Orv Hetil 2015; 156: 308-325. doi: 10.1556/ OH.2015.30062

11. Dubravcsik Z, Farkas G, Hegyi P, et al. Autoimmune pancreatitis. Evidence based management guidelines of the Hungarian Pancreatic Study Group. Orv Hetil 2015; 156: 292-307. doi: 10.1556/ OH.2015.30061

12. Takács T, Czakó L, Dubravcsik Z, et al. Chronic pancreatitis. Evidence based management guidelines of the Hungarian Pancreatic Study Group. Orv Hetil 2015; 156: 262-288. doi: 10.1556/OH.2015.30060 
13. Hritz I, Czakó L, Dubravcsik Z, et al. Acute pancreatitis. Evidence-based practice guidelines, prepared by the Hungarian Pancreatic Study Group Orv Hetil 2015; 156: 244-161. doi: 10.1556/OH.2015.30059

14. Dubravcsik Z, Madácsy L, Gyökeres T, et al. Preventive pancreatic stents in the management of acute biliary pancreatitis (PREPAST trial): pre-study protocol for a multicenter, prospective, randomized, interventional, controlled trial. Pancreatology 2015; 15: 115-123. doi: 10.1016/j.pan.2015.02.007

15. Hritz I, Hegyi P. Early Achievable Severity (EASY) index for simple and accurate expedite risk stratification in acute pancreatitis. J Gastrointestin Liver Dis 2015; 24: 177-182. doi: 10.15403/jgld.2014.1121.242.easy

16. Párniczky A, Mosztbacher D, Zsoldos F, et al; Hungarian Pancreatic Study Group and the International Association of Pancreatology. Analysis of Pediatric Pancreatitis (APPLE Trial): Pre-Study Protocol of a Multinational Prospective Clinical Trial. Digestion 2015; 93: 105-110. doi: $10.1159 / 000441353$

17. Zsoldos F, Párniczky A, Mosztbacher D, et al; Hungarian Pancreatic Study Group and the International Association of Pancreatology. Pain in the Early Phase of Pediatric Pancreatitis (PINEAPPLE Trial): PreStudy Protocol of a Multinational Prospective Clinical Trial. Digestion 2016; 93: 121-126. doi: 10.1159/000441352

18. Malvezzi M, Bertuccio P, Levi F, La Vecchia C, Negri E. European cancer mortality predictions for the year 2013. Ann Oncol 2013; 24: 792-800. doi: 10.1093/annonc/mdt010

19. Iodice S, Gandini S, Maisonneuve P, Lowenfels AB. Tobacco and the risk of pancreatic cancer: a review and meta-analysis. Langenbecks Arch Surg 2008; 393: 535-545. doi: 10.1007/s00423-007-0266-2

20. Balku E, Demjén T, Vámos M, Vitrai J. Survey for smoking in adults 2012. National Institute for Health Development, Budapest, 2012. Available at: http://www.fokuszpont.dohanyzasvisszaszoritasa.hu/ sites/default/files/17_evnel_idosebb_lakossag_dohanyzas_felmeres_ honlapra_20131009

21. Lowenfels AB, Maisonneuve P, Lankish PG. Chronic pancreatitis and the risk factors for pancreatic cancer. Gastroenterol Clin North Am 1999; 28: 673-685. doi: 10.1016/S0889-8553(05)70080-7

22. Ben Q, Xu M, Ning X, et al. Diabetes mellitus and risk of pancreatic cancer: a meta-analysis of cohort studies. Eur J Cancer 2011; 47 1928-1937. doi: 10.1016/j.ejca.2011.03.003

23. World Cancer Research Fund, American Institute for Cancer Research. Food Nutrition, Physiscal Activity, and the Prevention of Cancer: a Global Perspective. Washington DC; AICR, 2007.

24. Li D, Jiao L. Molecular epidemiology of pancreatic cancer. Int J Gastrointest Cancer 2003; 33: 3-14. doi: 10.1385/IJGC:33:1:3

25. Goonetilleke KS, Siriwardena AK. Systematic review of carbohydrate antigen (CA 19-9) as a biochemical marker in the diagnosis of pancreatic cancer. Eur J Surg Oncol 2007; 33: 266-270. doi: 10.1016/j.ejso.2006.10.004

26. Li D, Xie K, Wolff R, Abbruzzese JL. Pancreatic cancer. Lancet 2004 363: 1049-1057. doi: 10.1016/S0140-6736(04)15841-8

27. Hidalgo M, Cascinu S, Kleeff J, et al. Addressing the challenges of pancreatic cancer: future directions for improving outcomes. Pancreatology 2015; 15: 8-18. doi: 10.1016/j.pan.2014.10.001

28. Tol JA, van Hooft JE, Timmer R, et al. Metal or plastic stents for preoperative biliary drainage in resectable pancreatic cancer. Gut 2015. doi: 10.1136/gutjnl-2014-308762

29. Conroy T, Desseigne F, Ychou M, et al. FOLFIRINOX versus gemcitabine for metastatic pancreatic cancer. N Engl J Med 2011; 364: 1817-1825. doi: 10.1056/NEJMoa1011923

30. Von Hoff DD, Ervin T, Arena FP, et al. Increased Survival in Pancreatic Cancer with nab-Paclitaxel plus Gemcitabine. N Engl J Med 2013; 369 1691-1703. doi: 10.1056/NEJMoa1304369 